EPJ Web of Conferences 112,04006 (2016)

DOI: $10.1051 /$ epjconf/201611204006

(C) Owned by the authors, published by EDP Sciences, 2016

\title{
Forward di-jet production in dilute-dense collisions
}

\author{
E. Petreska ${ }^{1,2, a}$, P. Kotko ${ }^{3}$, K. Kutak ${ }^{4}$, C. Marquet ${ }^{1}$, S. Sapeta ${ }^{4,5}$, and A. van Hameren ${ }^{4}$ \\ ${ }^{1}$ Centre de Physique Théorique, École Polytechnique, CNRS, Université Paris-Saclay, F-91128 Palaiseau, \\ France \\ ${ }^{2}$ Departamento de Física de Partículas and IGFAE, Universidade de Santiago de Compostela, 15782 San- \\ tiago de Compostela, Spain \\ ${ }^{3}$ Department of Physics, Penn State University, University Park, 16803 PA, USA \\ ${ }^{4}$ The H. Niewodniczański Institute of Nuclear Physics PAN, Radzikowskiego 152, 31-342 Kraków, Poland \\ ${ }^{5} \mathrm{CERN} \mathrm{PH}-\mathrm{TH}, \mathrm{CH}-1211$, Geneva 23, Switzerland
}

\begin{abstract}
We derive a factorization formula for forward production of two jets in dilutedense collisions that is valid for an arbitrary value of the momentum imbalance of the jets, $k_{t}$. This generalizes the transverse momentum dependent (TMD) factorization formula that has been derived before by Dominguez et al. Their formula is valid only for small values of the transverse momentum of the small- $x$ gluon from the target; it has $k_{t}$ dependent TMD gluon distributions, but on-shell hard matrix elements. We extend the TMD formula to all ranges of $k_{t}$ by including off-shell matrix elements. We also add finite $N_{c}$ corrections. The new formula encompasses both, the TMD factorization for small $k_{t}$ on the order of the saturation scale, and the High Energy Factorization (HEF) for large $k_{t}$ on the order of the momentum of the jets. The TMD and HEF factorizations can be derived from the Color Glass Condensate (CGC) formula for forward di-jet production in the appropriate limits. We show explicitly the equivalence of HEF and CGC in the dilute target approximation.
\end{abstract}

\section{Introduction}

The production of two jets in the forward rapidity direction of ultra-relativistic proton-proton and proton-nucleus collisions is sensitive to the small- $x$ gluon distributions in the target, and the large$x$ partons in the projectile. The longitudinal momentum fraction $x$ of the partons is defined with respect to the momentum of the parent hadron. The wave function of the projectile is obtained from perturbative quantum chromodynamics (pQCD), while the gluon distributions in the target are in the non-linear saturation regime. Di-jet production in asymmetric collisions has been studied in different theoretical frameworks, with different ranges of values for the momentum imbalance.

The transverse momentum of the produced jets, $P_{t}$, is one of the hardest scales in the problem, while the saturation momentum, $Q_{s}$, is one of the softest scales. The third scale in this problem is the momentum imbalance of the jets (or equivalently the transverse momentum of the interacting gluon from the target), $k_{t}$. The color glass condensate (CGC) approach [1] does not assume any particular ordering of these scales, and in the most general case there is no $k_{t}$ factorization formula for the

a e-mail: elena.petreska@usc.es 
cross section. The transverse momentum dependent (TMD) factorization [2], [3] is valid when the momentum imbalance is close to $Q_{s}$, and both are much smaller than the hard momentum of the jets, $k_{t} \sim Q_{s} \ll P_{t}$. The high-energy factorization (HEF) [4] [5] describes the region of large $k_{t}$ values on the order of $P_{t}, Q_{s} \ll k_{t} \sim P_{t}$. We unify the different approaches by deriving a formula valid for the whole range of $k_{t}$ values between $Q_{s}$ and $P_{t}$ [6].

In section 2 we derive the HEF formula from a CGC calculation in the limit of a dilute target, by restricting the scattering to two gluon exchanges. In section 3 we revise the TMD factorization formula that was derived in Ref. [3] in the large- $N_{c}$ limit. We include all finite $N_{c}$ correction, introduce three new gluon distributions, and reduce the number of independent distributions to two per channel. We improve the finite- $N_{c}$ TMD factorization in section 4 by restoring the $k_{t}$ dependence in the matrix elements.

\section{Derivation of the high-energy factorization from the color glass condensate cross section in the dilute target limit}

The dilute projectile in the HEF formula for di-jet production is represented with a parton distribution function of collinear factorization, $f_{a / p}\left(x_{1}, \mu^{2}\right)$, the dense target with one $k_{t}$-dependent gluon distribution, $\mathcal{F}_{g / A}\left(x_{2}, k_{t}\right)$, and the hard part of the scattering with off-shell matrix elements, $\overline{\mathcal{M}_{a g^{*} \rightarrow c d}}$ [5], [7]:

$$
\frac{d \sigma^{p A \rightarrow \text { dijets }+X}}{d y_{1} d y_{2} d^{2} p_{1 t} d^{2} p_{2 t}}=\frac{1}{16 \pi^{3}\left(x_{1} x_{2} s\right)^{2}} \sum_{a, c, d} x_{1} f_{a / p}\left(x_{1}, \mu^{2}\right) \mid{\overline{\mathcal{M}_{a g^{*} \rightarrow c d}}}^{2} \mathcal{F}_{g / A}\left(x_{2}, k_{t}\right) \frac{1}{1+\delta_{c d}} .
$$

In the above expression $s$ is the center of mass energy squared, $p_{1 t}$ and $p_{2 t}$ are the transverse momenta of the outgoing particles, and $y_{1}$ and $y_{2}$ are their rapidities. The longitudinal momentum fractions of the parton from the projectile and the gluon from the target are $x_{1}$ and $x_{2}$, respectively. The matrix elements have been calculated in Refs. [5], [8] and [9]. The HEF formula is an ansatz formula which turns out to be valid for large $k_{t}$ values, with $\mathcal{F}_{g / A}\left(x_{2}, k_{t}\right)$ equal to the unintegrated gluon distribution entering in the cross section for deep inelastic scattering (DIS). This distribution is associated with the $S$-matrix for a quark-anitquark dipole scattering:

$$
\mathcal{F}_{g / A}\left(x_{2}, k_{t}\right)=\frac{N_{c}}{\alpha_{s}(2 \pi)^{3}} \int d^{2} \mathbf{v} d^{2} \mathbf{v}^{\prime} e^{-i k_{t} \cdot\left(\mathbf{v}-\mathbf{v}^{\prime}\right)} \nabla_{\mathbf{v}-\mathbf{v}^{\prime}}^{2}\left[1-S_{q \bar{q}}^{(2)}\left(\mathbf{v}, \mathbf{v}^{\prime}\right)\right],
$$

where $S_{q \bar{q}}^{(2)}\left(\mathbf{v}, \mathbf{v}^{\prime}\right)$ is a correlator of two fundamental Wilson lines,

$$
S_{q \bar{q}}^{(2)}\left(\mathbf{v}, \mathbf{v}^{\prime}\right)=\frac{1}{N_{c}}\left\langle\operatorname{Tr} U(\mathbf{v}) U^{\dagger}\left(\mathbf{v}^{\prime}\right)\right\rangle
$$

The Wilson lines are path ordered exponentials of the gauge field of the target, $A_{\mu}$, and here, we define them in the light-cone gauge $A^{+}=0$ of the projectile,

$$
U(\mathbf{x})=\mathcal{P} \exp \left[i g \int d x^{+} A_{a}^{-}\left(x^{+}, \mathbf{x}\right) t^{a}\right] .
$$

The multi-gluon scatterings of the projectile parton with the dense field of the target, in the CGC cross section, are described with more complicated objects than the dipole $S_{q \bar{q}}^{(2)}$. The cross section for the $q g^{*} \rightarrow q g$ channel, for example, was calculated in Ref. [10], and it was shown that it involves four-point and three-point correlators of fundamental and/or adjoint Wilson lines as well, describing 
the multiple scatterings of a quark and a gluon in the amplitude and its complex conjugate. The fourpoint correlator is an expectation value of two fundamental Wilson lines, $U$, and two adjoint Wilson lines, $V$, (all at different transverse coordinates):

$$
S_{q g \bar{q} g}^{(4)}\left(\mathbf{b}, \mathbf{x}, \mathbf{b}^{\prime}, \mathbf{x}\right)=1 / N_{c}\left\langle\operatorname{Tr}\left(U(\mathbf{b}) U^{\dagger}\left(\mathbf{b}^{\prime}\right) t^{d} t^{c}\right)\left[V(\mathbf{x}) V^{\dagger}\left(\mathbf{x}^{\prime}\right)\right]^{c d}\right\rangle_{x_{2}} .
$$

The three-point correlator involves two fundamental and one adjoint Wilson line:

$$
S_{q q \bar{q}}^{(3)}\left(\mathbf{b}, \mathbf{x}, \mathbf{z}^{\prime}\right)=1 / N_{c}\left\langle\operatorname{Tr}\left(U^{\dagger}\left(\mathbf{z}^{\prime}\right) t^{c} U(\mathbf{b}) t^{d}\right) V^{c d}(\mathbf{x})\right\rangle_{x_{2}} .
$$

In the dilute target approximation (a target with two scattering centres), the $S_{q g \bar{q} g}^{(4)}$ and $S_{q g \bar{q}}^{(3)}$ correlators can be written in terms of the dipole $S_{q \bar{q}}^{(2)}$, and they can be related to the gluon distribution $\mathcal{F}_{g / A}\left(x_{2}, k_{t}\right)$ from the HEF formula. This approximation amounts to expanding the Wilson lines to second order in the background field $A_{\mu}$. In this approximation $S_{q g \bar{q} q}^{(4)}$ and $S_{q q \bar{q}}^{(3)}$ are [6]:

$$
\begin{aligned}
S_{q q \bar{q} g}^{(4)}\left(\mathbf{b}, \mathbf{x}, \mathbf{b}^{\prime}, \mathbf{x}^{\prime}\right) & \simeq S^{(2)}\left(\mathbf{b}, \mathbf{b}^{\prime}\right)-\frac{C_{A}}{C_{F}}\left[1-S^{(2)}\left(\mathbf{x}, \mathbf{x}^{\prime}\right)\right] \\
& -\frac{C_{A}}{2 C_{F}}\left[S^{(2)}\left(\mathbf{x}^{\prime}, \mathbf{b}\right)+S^{(2)}\left(\mathbf{x}, \mathbf{b}^{\prime}\right)-S^{(2)}(\mathbf{x}, \mathbf{b})-S^{(2)}\left(\mathbf{x}^{\prime}, \mathbf{b}^{\prime}\right)\right], \\
S_{q q \bar{q}}^{(3)}\left(\mathbf{b}, \mathbf{x}, \mathbf{v}^{\prime}\right) & \simeq \frac{C_{A}}{2 C_{F}}\left[S^{(2)}(\mathbf{b}, \mathbf{x})+S^{(2)}\left(\mathbf{x}, \mathbf{v}^{\prime}\right)-\frac{1}{C_{A}^{2}} S^{(2)}\left(\mathbf{b}, \mathbf{v}^{\prime}\right)\right]-\frac{C_{A}}{C_{F}} .
\end{aligned}
$$

With the above results we express the CGC di-jet cross section only in terms of the DIS distribution $\mathcal{F}_{g / A}\left(x_{2}, k_{t}\right)$, and we reproduce the matrix elements $\overline{\mathcal{M}_{a g^{*} \rightarrow c d}}$ from the HEF formula exactly [6]. We derive the HEF formula from the CGC cross section for a dilute target for all three channels: $q g^{*} \rightarrow q g$, $g g^{*} \rightarrow q \bar{q}$ and $g g^{*} \rightarrow g g$.

\section{Simplified TMD factorization for finite $N_{c}$}

A TMD factorization formula for forward di-jet production for small $k_{t}$ values, $Q_{s} \ll k_{t} \sim P_{t}$, was derived in Ref. [3], in the large- $N_{c}$ limit. This is the scenario of nearly back-to-back di-jets. The target is described with five $k_{t}$ dependent gluon distributions, instead of one, while the $k_{t}$ dependence is not present in the matrix elements (the incoming gluon from the target is put on shell). The different TMD distributions in the cross section represent the resummation of collinear gluons from the target that couple to the hard part. The resummation depends on the color flow in the $2 \rightarrow 2$ sub-process, and brings different gauge link structure in the gluon densities for different $2 \rightarrow 2$ Feynman diagrams. The gauge links, products of such resummation, turn a generic correlator of gluon field strength tensors

$$
\mathcal{F}\left(x_{2}, k_{t}\right) \stackrel{\text { naive }}{=} 2 \int \frac{d \xi^{+} d^{2} \boldsymbol{\xi}}{(2 \pi)^{3} p_{A}^{-}} e^{i x_{2} p_{A}^{-} \xi^{+}-i k_{t} \cdot \xi}\left\langle A\left|\operatorname{Tr}\left[F^{i-}\left(\xi^{+}, \boldsymbol{\xi}\right) F^{i-}(0)\right]\right| A\right\rangle
$$

into several (different) gauge invariant TMD gluon distributions that will emerge in the factorized cross section. The TMD's for all types of $2 \rightarrow 2$ diagrams have been calculated in Ref. [2].

We revise the derivation of the TMD formula for this process, keeping $N_{c}$ finite. For each hard factor from Ref. [3] we identify the corresponding gluon distributions from Ref. [2], for all three channels. The finite $N_{c}$ corrections bring three new TMD distributions compared to the large- $N_{c}$ case, as well as corrections to the hard parts that were previously omitted. The TMD gluon distributions are: 


$$
\begin{aligned}
& \mathcal{F}_{q g}^{(1)}=\int \frac{2 d \xi^{+} d^{2} \xi}{(2 \pi)^{3} p_{A}^{-}} e^{i x_{2} p_{A}^{-} \xi^{+}-i k_{t} \cdot \xi}\left\langle\operatorname{Tr}\left[F(\xi) \mathcal{U}^{[-]^{\dagger}} F(0) \mathcal{U}^{[+]}\right]\right\rangle, \\
& \left.\mathcal{F}_{q g}^{(2)}=\int \frac{2 d \xi^{+} d^{2} \xi}{(2 \pi)^{3} p_{A}^{-}} e^{i x_{2} p_{A}^{-} \xi^{+}-i k_{t} \cdot \xi} \operatorname{Tr}\left[F(\xi) \frac{\operatorname{Tr}\left[\mathcal{U}^{[\square]}\right]}{N_{c}} \mathcal{U}^{[+] \dagger} F(0) \mathcal{U}^{[+]}\right]\right\rangle \\
& \left.\mathcal{F}_{g g}^{(1)}=\int \frac{2 d \xi^{+} d^{2} \xi}{(2 \pi)^{3} p_{A}^{-}} e^{i x_{2} p_{A}^{-} \xi^{+}-i k_{t} \cdot \xi} \operatorname{Tr}\left[F(\xi) \frac{\operatorname{Tr}\left[\mathcal{U}^{[\square]}\right]}{N_{c}} \mathcal{U}^{[-] \dagger} F(0) \mathcal{U}^{[+]}\right]\right\rangle, \\
& \mathcal{F}_{g g}^{(2)}=\int \frac{2 d \xi^{+} d^{2} \xi}{(2 \pi)^{3} p_{A}^{-}} e^{i x_{2} p_{A}^{-} \xi^{+}-i k_{t} \cdot \xi} \frac{1}{N_{c}}\left\langle\operatorname{Tr}\left[F(\xi) \mathcal{U}^{[\square] \dagger}\right] \operatorname{Tr}\left[F(0) \mathcal{U}^{[\square]}\right]\right\rangle, \\
& \mathcal{F}_{g g}^{(3)}=\int \frac{2 d \xi^{+} d^{2} \xi}{(2 \pi)^{3} p_{A}^{-}} e^{i x_{2} p_{A}^{-} \xi^{+}-i k_{t} \cdot \xi}\left\langle\operatorname{Tr}\left[F(\xi) \mathcal{U}^{[+] \dagger} F(0) \mathcal{U}^{[+]}\right]\right\rangle, \\
& \mathcal{F}_{g g}^{(4)}=\int \frac{2 d \xi^{+} d^{2} \xi}{(2 \pi)^{3} p_{A}^{-}} e^{i x_{2} p_{A}^{-} \xi^{+}-i k_{t} \cdot \xi}\left\langle\operatorname{Tr}\left[F(\xi) \mathcal{U}^{[-] \dagger} F(0) \mathcal{U}^{[-]}\right]\right\rangle, \\
& \mathcal{F}_{g g}^{(5)}=\int \frac{2 d \xi^{+} d^{2} \xi}{(2 \pi)^{3} p_{A}^{-}} e^{i x_{2} p_{A}^{-} \xi^{+}-i k_{t} \cdot \xi}\left\langle\operatorname{Tr}\left[F(\xi) \mathcal{U}^{[\square] \dagger} \mathcal{U}^{[+] \dagger} F(0) \mathcal{U}^{[\square]} \mathcal{U}^{[+]}\right]\right\rangle \\
& \mathcal{F}_{g g}^{(6)}=\int \frac{2 d \xi^{+} d^{2} \xi}{(2 \pi)^{3} p_{A}^{-}} e^{i x_{2} p_{A}^{-} \xi^{+}-i k_{t} \cdot \xi}\left\langle\operatorname{Tr}\left[F(\xi) \mathcal{U}^{[+] \dagger} F(0) \mathcal{U}^{[+]}\right]\left(\frac{\operatorname{Tr}\left[\mathcal{U}^{[\square]}\right]}{N_{c}}\right)^{2}\right\rangle .
\end{aligned}
$$

The new distributions are $\mathcal{F}_{g g}^{(3-5)}$. The gauge links are defined as $\mathcal{U}^{[ \pm]}=U(0, \pm \infty ; \mathbf{0}) U\left( \pm \infty, \xi^{+} ; \xi\right)$, and $\mathcal{U}^{[\square]}=\mathcal{U}^{[+]} \mathcal{U}^{[-] \dagger}=\mathcal{U}^{[-]} \mathcal{U}^{[+] \dagger}$, where $U(a, b ; \mathbf{x})=\mathcal{P} \exp \left[i g \int_{a}^{b} d x^{+} A_{a}^{-}\left(x^{+}, \mathbf{x}\right) t^{a}\right]$. The gluon distribution $\mathcal{F}_{q g}^{(1)}$ is the dipole distribution, and $\mathcal{F}_{g g}^{(3)}$ is the Weizsäcker-Williams gluon distribution.

Not all of the matrix elements that correspond to the above distributions are independent. We reduce the number of independent matrix elements and their corresponding distributions to two per channel, and we write a more compact TMD factorization formula for forward di-jet production at finite $N_{c}$ [6]:

$$
\frac{d \sigma^{p A \rightarrow \mathrm{dijets}+X}}{d^{2} P_{t} d^{2} k_{t} d y_{1} d y_{2}}=\frac{\alpha_{s}^{2}}{\left(x_{1} x_{2} s\right)^{2}} \sum_{a, c, d} x_{1} f_{a / p}\left(x_{1}, \mu^{2}\right) \sum_{i=1}^{2} K_{a g \rightarrow c d}^{(i)} \Phi_{a g \rightarrow c d}^{(i)}\left(k_{t}\right) \frac{1}{1+\delta_{c d}} .
$$

The new hard factors, $K_{a g \rightarrow c d}^{(i)}$, are given in Table 1. The respective TMD's are linear combinations of $\mathcal{F}_{a g}^{(i)}$. For the $q g \rightarrow q g$ channel:

$$
\Phi_{q g \rightarrow q g}^{(1)}=\mathcal{F}_{q g}^{(1)} \quad \text { and } \quad \Phi_{q g \rightarrow q g}^{(2)}=\frac{1}{\left(N_{c}^{2}-1\right)}\left(-\mathcal{F}_{q g}^{(1)}+N_{c}^{2} \mathcal{F}_{q g}^{(2)}\right) .
$$

For the $g g \rightarrow q \bar{q}$ channel:

$$
\Phi_{g g \rightarrow q \bar{q}}^{(1)}=\frac{1}{\left(N_{c}^{2}-1\right)}\left(N_{c}^{2} \mathcal{F}_{g g}^{(1)}-\mathcal{F}_{g g}^{(3)}\right) \quad \text { and } \quad \Phi_{g g \rightarrow q \bar{q}}^{(2)}=-N_{c}^{2} \mathcal{F}_{g g}^{(2)}+\mathcal{F}_{g g}^{(3)}
$$

For the $g g \rightarrow g g$ channel:

$$
\begin{aligned}
& \Phi_{g g \rightarrow g g}^{(1)}=\frac{1}{2 N_{c}^{2}}\left(N_{c}^{2} \mathcal{F}_{g g}^{(1)}-2 \mathcal{F}_{g g}^{(3)}+\mathcal{F}_{g g}^{(4)}+\mathcal{F}_{g g}^{(5)}+N_{c}^{2} \mathcal{F}_{g g}^{(6)}\right) \quad \text { and } \\
& \Phi_{g g \rightarrow g g}^{(2)}=\frac{1}{2 N_{c}^{2}}\left(N_{c}^{2} \mathcal{F}_{g g}^{(2)}-2 \mathcal{F}_{g g}^{(3)}+\mathcal{F}_{g g}^{(4)}+\mathcal{F}_{g g}^{(5)}+N_{c}^{2} \mathcal{F}_{g g}^{(6)}\right) .
\end{aligned}
$$


Table 1. The hard factors $K_{a g \rightarrow c d}^{(i)}$ for an on-shell gluon accompanying the gluon TMDs $\Phi_{a g \rightarrow c d}^{(i)}$. The Mandelstam variables are defined as $\hat{s}=\left(p_{1}+p_{2}\right)^{2}, \hat{t}=\left(p_{1}-k\right)^{2}$ and $\hat{u}=\left(p_{2}-k\right)^{2}$.

\begin{tabular}{c|c|c}
\hline & $K_{a g \rightarrow c d}^{(1)}$ & $K_{a g \rightarrow c d}^{(2)}$ \\
\hline 1 & $-\frac{C_{F}}{N_{c}} \frac{\hat{s}\left(\hat{s}^{2}+\hat{u}^{2}\right)}{\hat{t}^{2} \hat{u}}$ & $-\frac{\hat{s}^{2}+\hat{u}^{2}}{2 \hat{t}^{2} \hat{s} \hat{u}}\left[\hat{u}^{2}+\frac{\hat{s}^{2}-\hat{t}^{2}}{N_{c}^{2}}\right]$ \\
\hline 2 & $\frac{1}{2 N_{c}} \frac{\left(\hat{t}^{2}+\hat{u}^{2}\right)^{2}}{\hat{s}^{2} \hat{t} \hat{u}}$ & $-\frac{1}{2 C_{F} N_{c}^{2}} \frac{\hat{t}^{2}+\hat{u}^{2}}{\hat{s}^{2}}$ \\
\hline 3 & $\frac{2 N_{c}}{C_{F}} \frac{\left(\hat{s}^{2}-\hat{t} \hat{u}\right)^{2}\left(\hat{t}^{2}+\hat{u}^{2}\right)}{\hat{t}^{2} \hat{u}^{2} \hat{s}^{2}}$ & $\frac{2 N_{c}}{C_{F}} \frac{\left(\hat{s}^{2}-\hat{t} \hat{u}\right)^{2}}{\hat{t} \hat{u} \hat{s}^{2}}$ \\
\hline
\end{tabular}

Table 2. The hard factors $K_{a g^{*} \rightarrow c d}^{(i)}$ for an off-shell gluon accompanying the gluon TMDs $\Phi_{a g \rightarrow c d}^{(i)}$. The bared versions of the Mandelstam variables are defined as $\bar{s}=\left(x_{2} p_{A}+p\right)^{2}, \bar{t}=\left(x_{2} p_{A}-p_{1}\right)^{2}$ and $\bar{u}=\left(x_{2} p_{A}-p_{2}\right)^{2}$. We have also denoted $v=\bar{s}^{4}+\bar{t}^{4}+\bar{u}^{4}$ and $w=\bar{u} \hat{u}+\bar{t} \hat{t}-\bar{s} \hat{s}$.

\begin{tabular}{c|c|c}
\hline & $K_{a g^{*} \rightarrow c d}^{(1)}$ & $K_{a g^{*} \rightarrow c d}^{(1)}$ \\
\hline 1 & $-\frac{\bar{u}\left(\bar{s}^{2}+\bar{u}^{2}\right)}{2 \bar{t} \hat{t} \hat{s}}\left(1+\frac{\bar{s} \hat{s}-\bar{t} \hat{t}}{N_{c}^{2} \bar{u} \hat{u}}\right)$ & $-\frac{C_{F}}{N_{c}} \frac{\bar{s}\left(\bar{s}^{2}+\bar{u}^{2}\right)}{\bar{t} \hat{t} \hat{u}}$ \\
\hline 2 & $\frac{1}{2 N_{c}} \frac{\left(\bar{t}^{2}+\bar{u}^{2}\right)(\bar{u} \hat{u}+\bar{t} \hat{t})}{\bar{s} \hat{s} \hat{t} \hat{u}}$ & $\frac{\left(\bar{t}^{2}+\bar{u}^{2}\right) w}{4 N_{c}^{2} C_{F} \bar{s} \hat{s} \hat{t} \hat{u}}$ \\
\hline 3 & $\frac{N_{c}}{C_{F}} \frac{v(\bar{u} \hat{u}+\bar{t} \hat{t})}{\bar{t} \hat{t} \hat{u} \hat{u} \bar{s} \hat{s}}$ & $-\frac{N_{c}}{2 C_{F}} \frac{v w}{\bar{t} \hat{t} \hat{u} \hat{u} \hat{s} \hat{s}}$ \\
\hline
\end{tabular}

\section{Unifying TMD factorization formula}

As we mention in the previous section, the matrix elements in the TMD factorization were derived for an on-shell incoming gluon from the target, which limits the applicability of the formula to small values of $k_{t}$. We propose a solution to this limitation by including off-shell matrix elements in the factorization formula and by restoring the $k_{t}$ dependence in the hard part. We calculate the $k_{t}$ dependent matrix elements with two methods. The high energy factorization approach is a Feynman diagram based calculation, with light-cone gauge for the on-shell gluons, with the gauge vector $n$ set to be equal to the four momentum of the target, $n=p_{A}$, and with prescribing a longitudinal polarization vector to the off-shell gluon from the target of the form $\epsilon_{\mu}^{0}=i \sqrt{2} x_{2} p_{A \mu} /\left|k_{t}\right|$ [4]. The advantage of this particular prescription is that it generates gauge invariant matrix elements, but the drawback is that the gauge invariance is not clearly manifested for the separate Feynman diagrams. The second method, the helicity method for TMD amplitudes [11] [12], reveals the gauge invariance already on the level of the amplitudes. These are the so-called color ordered amplitudes that represent the coefficients of a color decomposition of a generic amplitude (involving an arbitrary number of gluons and/or quarks) into a color part and a kinematic part. The color ordered amplitudes are functions of kinematic arguments only, and are gauge invariant. They give the hard factors, while the color part of the decomposition, after squaring, indicates the corresponding gluon TMDs. In addition, this pro- 
cedure takes advantage of the relations between the coefficients, removes the dependent hard factors from the beginning, and results in a compact factorization formula without redundancy.

We derive a factorization formula, similar to the one derived in the previous section, Eq. (10), but now with $k_{t}$ dependent matrix elements $K_{a g^{*} \rightarrow c d}^{(i)}\left(k_{t}\right)$ [6]. We apply both of the methods described above independently. The new formula is:

$$
\frac{d \sigma^{p A \rightarrow \text { dijets }+X}}{d^{2} P_{t} d^{2} k_{t} d y_{1} d y_{2}}=\frac{\alpha_{s}^{2}}{\left(x_{1} x_{2} s\right)^{2}} \sum_{a, c, d} x_{1} f_{a / p}\left(x_{1}, \mu^{2}\right) \sum_{i=1}^{2} K_{a g^{*} \rightarrow c d}^{(i)}\left(k_{t}\right) \Phi_{a g \rightarrow c d}^{(i)}\left(k_{t}\right) \frac{1}{1+\delta_{c d}},
$$

with the matrix elements given in Table 2. The above formula unifies the HEF approach to forward dijet production with the TMD factorization by establishing a framework applicable for hard jets, $P_{t} \gg Q_{s}$, but arbitrary $k_{t}$, and it is the main result of this work.

For phenomenological applications to di-jet azimuthal correlations in high-energy collisions, we study the new framework at large $N_{c}$, and with analytical models for the gluon distributions. We calculate the gluon densities that survive the large $N_{c}$ limit in the Golec-Biernat-Wusthoff model [13]:

$$
\begin{aligned}
& \mathcal{F}_{q g}^{(1)}\left(x_{2}, k_{t}\right)=\frac{N_{c} S_{\perp}}{2 \pi^{3} \alpha_{s}} \frac{S_{\perp}}{Q_{s}^{2}\left(x_{2}\right)} k_{t}^{2} \exp \left[-\frac{k_{t}^{2}}{Q_{s}^{2}\left(x_{2}\right)}\right], \\
& \mathcal{F}_{q g}^{(2)}\left(x_{2}, k_{t}\right)=\frac{N_{c} S_{\perp}}{2 \pi^{3} \alpha_{s}} \exp \left[-\frac{k_{t}^{2}}{Q_{s}^{2}\left(x_{2}\right)}\right] \int_{1}^{\infty} \frac{d t}{t(t+2)} \exp \left[\frac{2 k_{t}^{2}}{(t+2) Q_{s}^{2}\left(x_{2}\right)}\right], \\
& \mathcal{F}_{g g}^{(1)}\left(x_{2}, k_{t}\right)=\frac{N_{c} S_{\perp}}{16 \pi^{3} \alpha_{s}} \exp \left[-\frac{k_{t}^{2}}{2 Q_{s}^{2}\left(x_{2}\right)}\right]\left(2+\frac{k_{t}^{2}}{Q_{s}^{2}\left(x_{2}\right)}\right), \\
& \mathcal{F}_{g g}^{(2)}\left(x_{2}, k_{t}\right)=\frac{N_{c} S_{\perp}}{16 \pi^{3} \alpha_{s}} \exp \left[-\frac{k_{t}^{2}}{2 Q_{s}^{2}\left(x_{2}\right)}\right]\left(2-\frac{k_{t}^{2}}{Q_{s}^{2}\left(x_{2}\right)}\right), \\
& \mathcal{F}_{g g}^{(6)}\left(x_{2}, k_{t}\right)=\frac{N_{c} S_{\perp}}{4 \pi^{3} \alpha_{s}} \exp \left[-\frac{k_{t}^{2}}{2 Q_{s}^{2}\left(x_{2}\right)}\right] \int_{1}^{\infty} \frac{d t}{t(t+1)} \exp \left[\frac{k_{t}^{2}}{2(t+1) Q_{s}^{2}\left(x_{2}\right)}\right] .
\end{aligned}
$$

In the above expressions $S_{\perp}$ is the transverse area of the target. We also obtain their perturbative behaviour at large $k_{t}$ in the McLerran-Venugopalan model [14]. We derive the leading order term in $Q_{s}^{2} / k_{t}^{2}$, and we find that all of them scale as

$$
\simeq \frac{N_{c} S_{\perp} Q_{s}^{2}\left(x_{2}\right)}{4 \pi^{3} \alpha_{s} k_{t}^{2}}+O\left(\frac{Q_{s}^{4}\left(x_{2}\right)}{k_{t}^{4}} \log \frac{k_{t}^{2}}{\Lambda^{2}}\right)
$$

except $\mathcal{F}_{g g}^{(2)}$ that goes to zero. The above expressions for the densities, as well as numerical results that implement small- $x$ evolution, will be used for phenomenological application of the unifying formula in a forthcoming publication.

\section{Acknowledgments}

EP thanks the organizers of the Physics Opportunities at an Electron-Ion Collider 2015 conference for the invitation for this talk.

\section{References}

[1] F. Gelis, E. Iancu, J. Jalilian-Marian and R. Venugopalan, Ann. Rev. Nucl. Part. Sci. 60 (2010) 463. 
[2] C. J. Bomhof, P. J. Mulders and F. Pijlman, Eur. Phys. J. C 47 (2006) 147.

[3] F. Dominguez, C. Marquet, B. -W. Xiao and F. Yuan, Phys. Rev. D 83 (2011) 105005.

[4] S. Catani, M. Ciafaloni and F. Hautmann, Nucl. Phys. B 366 (1991) 135.

[5] M. Deak, F. Hautmann, H. Jung and K. Kutak, JHEP 0909 (2009) 121.

[6] P. Kotko, K. Kutak, C. Marquet, E. Petreska, S. Sapeta and A. van Hameren, JHEP 1509 (2015) 106

[7] K. Kutak and S. Sapeta, Phys. Rev. D 86 (2012) 094043.

[8] A. van Hameren, P. Kotko and K. Kutak, JHEP 1212 (2012) 029.

[9] A. van Hameren, P. Kotko and K. Kutak, JHEP 1301 (2013) 078.

[10] C. Marquet, Nucl. Phys. A 796 (2007) 41.

[11] M. L. Mangano and S. J. Parke, Phys. Rept. 200 (1991) 301.

[12] L. J. Dixon, arXiv:1310.5353 [hep-ph].

[13] K. J. Golec-Biernat and M. Wusthoff, Phys. Rev. D 59, (1998) 014017

[14] L. D. McLerran and R. Venugopalan, Phys. Rev. D 49, 2233 (1994), Phys. Rev. D 49, 3352 (1994); 